\title{
Design and Implementation of Long-Term Single-Lead ECG Monitor
}

\author{
Meng Shen, Shijing Xue \\ National Mobile Communications Research Laboratory, Southeast University, Nanjing, China \\ Email: shenmeng milink@163.com \\ Received January 2015
}

\begin{abstract}
Some heart diseases need long-term monitoring to diagnose. In this paper, we present a wearable single lead ECG monitoring device with low power consumption based on MSP430 and single-lead ECG front-end AD8232, which could acquire and store patient's ECG data for 7 days continuously. This device is available for long-term wearing with a small volume. Also, it could detect user's motion status with an acceleration sensor and supports Bluetooth 4.0 protocol. So it could be expanded to be a dynamic heart rate monitor and/or sleep quality monitor combined with smart phone. The device has huge potential of application for health care of human daily life.
\end{abstract}

\section{Keywords}

Single Lead ECG, Long-Term Monitor, Low Power Consumption

\section{Introduction}

Long term ECG monitoring has a great significance to the clinical diagnosis of heart disease. Some diseases have the characteristics of sporadic and transient in ECG performance. Usually, the current Holter monitors can only monitor patient's ECG for 24 hours continuously, unable to meet needs. For an example, 24-hour Holter monitor follow-up could not provide accurate heart rhythm status after surgical atrial fibrillation ablation therapy [1]. Long term monitoring device needs to be portable, comfortable and has no interference on the user's daily life. In recent years, many scholars and research institutions have designed many kinds of long-term ECG monitor devices, in order to record user's long-term ECG signals in daily activities. S. Suave Lobodzinski et al. presented a wearable long-term 14-day patch ECG monitor that was attached directly to the skin and required no electrodes and wires [2].

This paper presents a long-term single lead ECG monitoring device which uploads ECG data to computer through USB communication protocol and doing data analysis on computer. The system design is introduced in Section II, and software design is described in Section III. Section IV covers the experimental resaults and Section $\mathrm{V}$ is the conclusion.

\section{System Design}

\subsection{Design Considerations \& Specifications}

The ECG monitor realizes long-term single lead ECG monitoring and motion data acquisition, also supporting

How to cite this paper: Shen, M. and Xue, S.J. (2015) Design and Implementation of Long-Term Single-Lead ECG Monitor. Journal of Biosciences and Medicines, 3, 18-23. http://dx.doi.org/10.4236/jbm.2015.34003 
wireless transmission with computer and mobile phone, specifically including the following functions: 1) Single lead ECG signal acquisition with sampling frequency $256 \mathrm{~Hz}$ and sampling precision 12 bit; 2) Motion status monitoring with 3D acceleration sensor with sampling frequency $64 \mathrm{~Hz}$ and sampling precision 16 bit; 3) Storage of 7 days ECG data and motion data; 4) Support of USB interfaces, through which upload data to computer; 5) Support of Bluetooth wireless communication, capable of communicating with mobile phone wirelessly.

The system is used for long-term data acquisition and storage, so low-power design should be taken into consideration. Also, system must be small in volume and comfortable to be worn with no inference to user's daily life.

\subsection{System Architecture}

Since the device is mainly used to record ECG and motion data which doesn't involve in complex operations, single-chip microcomputer(SCM )is implemented in our system. Take the size and power comsuption into consideration, we choose MSP430F5524, a ultralow-power microcontroller of Texas Instrument Co. This chip is configured with integrated USB and PHY supporting USB 2.0, a high-performance 12-bit analog-to-digital converter (ADC), two universal serial communication interfaces (USCI) which can be used as SPI, IIC and UART respectively [3].

The system consists of the following modules MCU control module, ECG analog front end, Bluetooth module, SD card storage module and motion sensor module, power management module. The system architecture of device is shown in Figure 1.

A fully integrated single lead ECG front-end AD8232 form ADI company is adopted for ECG acquisition. ST company's three axis acceleration sensor is selected as motion sensor. Removable SD card is chosen as the ECG data storage unit, with a memory size of 4 GB, capable of 7 days' storage of patient's ECG data continuously. MSP430 communicate with SD card through SPI communication interface, implementation of ECG data's read and write, and realize ECG data' upload to computer based on USB communication protocol. Also, MCU communicate with Bluetooth module CC2540 through serial port UART, realizing the communication between monitor and mobile phone. USB interface is supported directly by MSP430F5524.

\subsection{ECG Acquisition}

We use a fully integrated single lead ECG front-end AD8232 with low-power. This device is designed for extract, amplify, and filter small biopotential signal in the presence of noisy conditions, such as those created by motion or remote electrode placement. It consists of a specialized instrumentation amplifier (IA), an operational amplifier (A1), a right leg drive amplifier (A2), and a midsupply reference buffer (A3). The AD8232 contains a specialized instrumentation amplifier that amplifies the ECG signal while rejecting the electrode half-cell potential on the same stage. This is possible with an indirect current feedback architecture, which reduces size and power compared with traditional implementations. In addition, the AD8232 includes leads off detection circuitry and an automatic fast restore circuit that brings back the signal shortly after leads are reconnected [4].

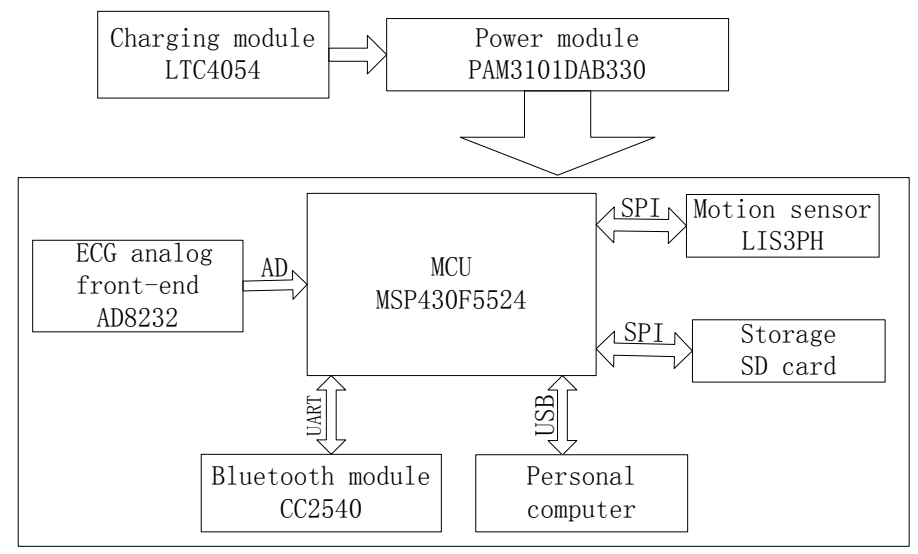

Figure 1. System architecture of ECG monitor. 
AD8232 is integrated with two-pole adjustable high-pass filter and three-pole low-pass filter, and right leg driven amplifier, providing convenient debugging interface.

\subsection{Motion Sensor}

The motion of human body in daily activities will bring interference to ECG signals [5]. In order to monitor human body's motion in daily activities, the system is equipped with acceleration sensor. LIS3DH chip from ST Company is selected as the acceleration sensor, which can output three axis acceleration of digital signal, and communicate with MSP430 through SPI interface.

\subsection{Wireless Transmission}

The wireless communication module choose the low power Bluetooth chip CC2540 form TI [6], communicating with MSP430 through the internal integrated UART, realizing wireless communication with mobile phone.

\subsection{Low Power Consumption Design}

The wearable ECG monitor device is used for the long-term ECG signal acquisition and storage of patients in daily life, so low power consumption design is important in system design.

Based on the realization of low power consumption, the system uses the following points: 1) Choose the ultralow-power microcontrollers MSP430F5524 form TI as our system' MCU; 2) Adopt the low-power, true system-on-chip Bluetooth CC2540 to realize wireless transmission; 3) The whole system adopts $3.3 \mathrm{~V}$ single power supply which powered by rechargeable lithium batteries. The battery voltage ranges from $3.7 \mathrm{~V}$ to $4.2 \mathrm{~V}$ and output constant voltage $3.3 \mathrm{~V}$ through a linear regulator CMOS PAM3101.

\section{Software Design}

The ECG monitor realizes continuous acquisition and storage of ECG signals and supports USB interface, through which enables data communications with computer.

\subsection{Main Program Design of MCU}

When device is power on, main program implements the initialization of AD converter, timer, SPI, USB and serial port modules first and then enable interrupt, entering the main loop program. In the loop program, USB interface state and electrode state are visited alternatively. When the device is connected to computer, device is accessed as a USB mass storage device class (MSC) and files in SD card is available directly by software of computer. The voltage of pin changes when electrode contact with the skin is detected. Once detection the voltage changes of pin, timer and AD converter start to work. The master flowchart is shown in Figure 2.

\subsection{Interrupt Service Routine}

The acquisition and storage of ECG data and motion data are implemented in the timer interrupt service routine. Interrupt service program flow chart is shown in Figure 3. The sampling frequency of ECG signal is $256 \mathrm{~Hz}$. However, motion signal's sampling frequency is set to $64 \mathrm{~Hz}$, which means motion signal will be sampled after four ECG signal samplings. Sampling data will be cached in a data array with a length of $512 \times 2$ bytes. When the preceding 512 bytes block (block 1) finished, data will be written to SD card files as a data packet and the following 512 bytes block (block 2) keeps caching sampling data. When the following 512 bytes block (block 2) finished, sampling data will be written to SD card in the same way. The use of $512 \times 2$ bytes array separates the process of sampling and storage, keeping the continuity of storage data.

\subsection{Management of SD Card Storage}

In this system, FAT32 file system is adopted for storage management. In SD card, cluster is defined as the storage unit formed by 8 sectors with 512 bytes per sector. To avoid user's wrong operations to files on SD card, functions of file creation and deletion are shielded in the SCSI Protocol layer from peripherals.

A new file is created in the root directory of the FAT32and opened when the device start to collect data. In the monitoring process, ECG data and motion data are written to the file as a data packet with length of 512 bytes in 


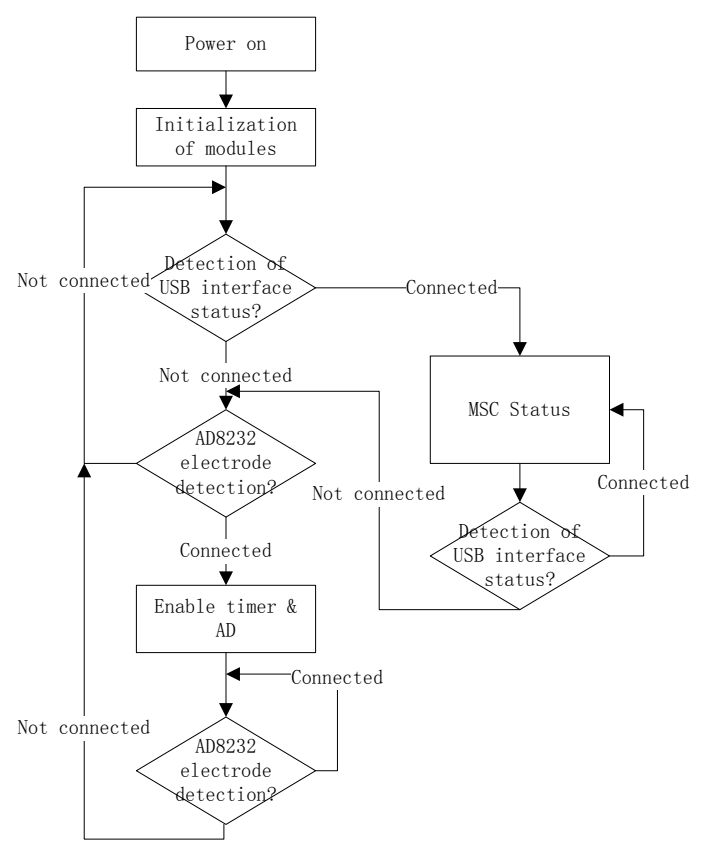

Figure 2. Master flowchart.

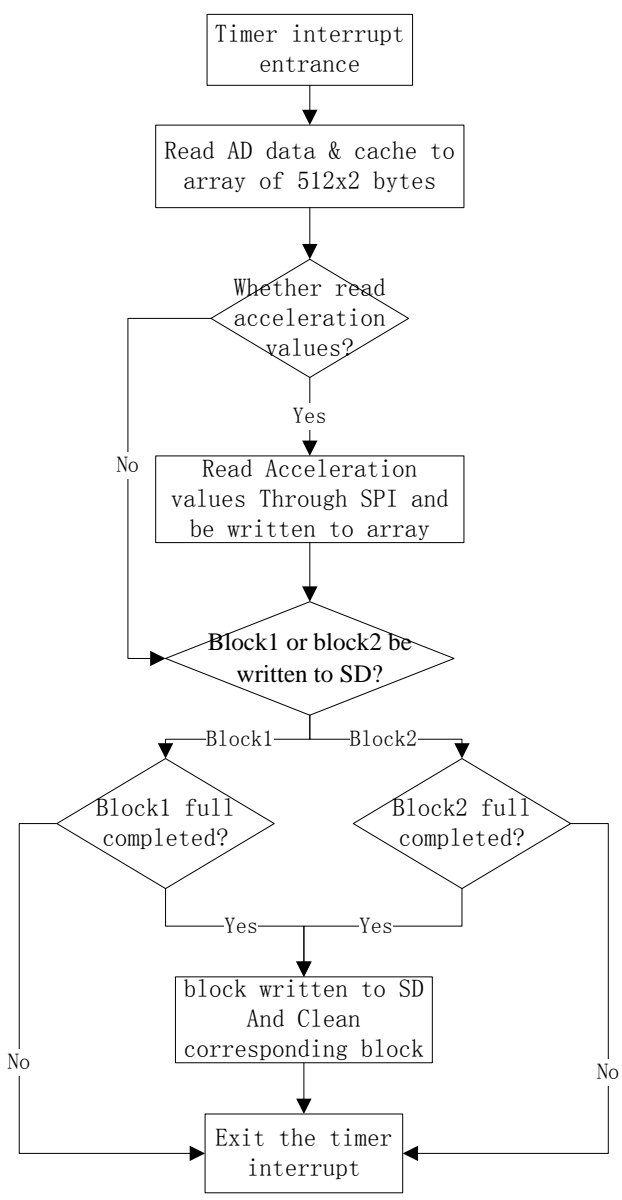

Figure 3. Interrupt service routine flowchart. 
binary code. The file is closed after the last data packet is written when the device stops monitor. Data structure is shown in Figure 4.

\subsection{USB Communication}

This device adopts MSC communication protocol (namely the USB mass storage device class) to realize data transmission between device and the computer. MSC protocol is a transport protocol used between computer and mobile device.

The device will be identified as MSC equipment when device is connected to the computer. MCU MSP430F5524 adopted in our system can work in the highest frequency of $20 \mathrm{MHz}$. In order to improve the transmission speed, SCSI command task is adjusted to the highest priority and only the system clock and statistical task are allowed. Finally, the data transfer speed reaches $250 \mathrm{~KB}$ per second.

\section{Experimental Results}

The volume of device is $30 \times 30 \times 6 \mathrm{~mm}$ (about one $¥$ coin size), with weight $12 \mathrm{~g}$, very small and light, comfortable to be worn. The system photo is shown as Figure 5.

The system was tested (ECG monitor worn in the chest near the heart) and the ECG signal is shown in Figure 6. As can be seen from the chart, the ECG waveforms maintain good character of ECG signals. P, QRS and T wave can be recognized easily, able to be used as clinical medical data analysis.

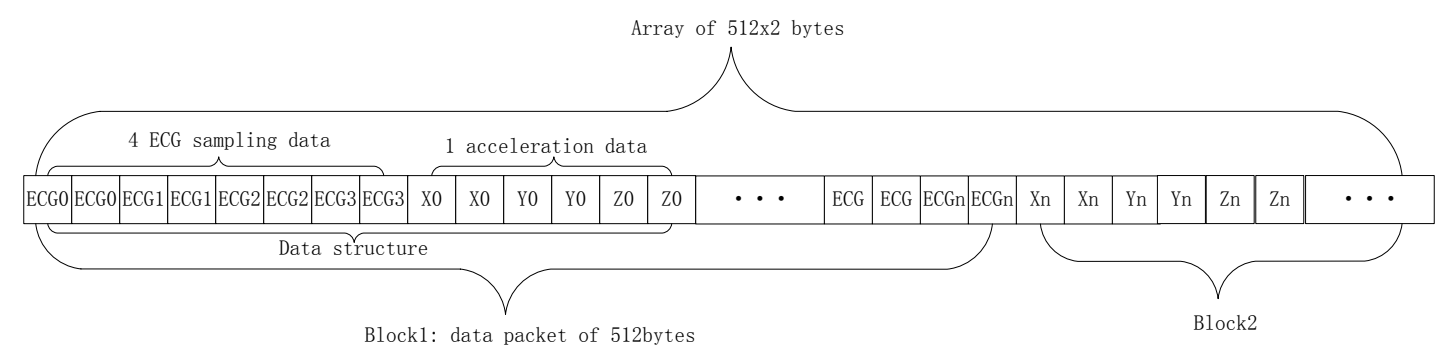

Figure 4. Data structure in SD card.

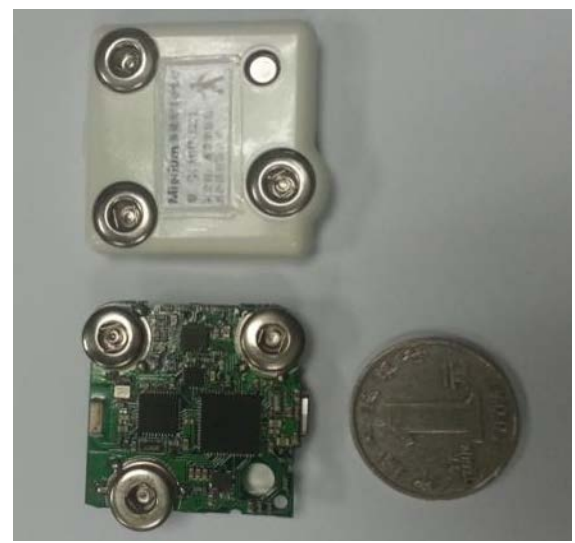

Figure 5. Picture of ECG monitor.

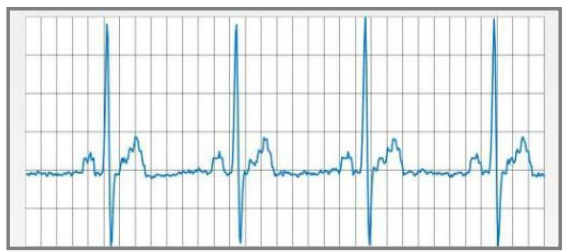

Figure 6. Display of ECG waveform. 
Our system has been clinically tested and is asking for the SFDA certification, can be used as a medicinal product on 7 days' single lead ECG screening.

\section{Conclusion}

In this paper, we present a wearable single lead ECG monitoring device with low power consumption based on MSP430 and single-lead ECG front-end AD8232, which could monitor and store patient's ECG data for 7 days continuously. This device is very convenient for long-term wearing with a small volume. Also, this system is equipped with an acceleration sensor and supports Bluetooth 4.0 protocol, could realizing patient's dynamic heart rate monitor and sleep quality analysis combined with mobile phone, which is suitable for mobile health and has a huge potential of application.

\section{References}

[1] Hanke, T., Charitos, E.I., Stierle, U., et al. (2009) Twenty-Four-Hour Holter Monitor Follow-Up Does Not Provide Accurate Heart Rhythm Status after Surgical Atrial Fibrillation Ablation Therapy: Up to 12 Months Experience with a Novel Permanently Implantable Heart Rhythm Monitor Device. Circulation, 120, S177-S184. http://dx.doi.org/10.1161/CIRCULATIONAHA.108.838474

[2] Lobodzinski, S.S. and Laks, M.M. (2012) New Devices for Very Long-Term ECG Monitoring. Cardiology Journal, 19, 210-214. http://dx.doi.org/10.5603/CJ.2012.0039

[3] Reid, W. (1997) Mixed-Signal Microcontroller. Texas Instruments. http://www.ti.com.cn/cn/lit/ds/symlink/msp430f5524.pdf

[4] Analog Devices, Inc. (2012) “AD8232” Rev. A. http://www.ntomsk.ru/uploads/datasheets/AD8232.pdf

[5] Yoon, S., Lee, S., Yun, Y., et al. (2007) A Development of Motion Artifacts Reduction Algorithm for ECG Signal in Textile Wearable Sensor. World Congress on Medical Physics and Biomedical Engineering 2006, Springer, Berlin, 1210-1213.

[6] Texas Instruments (2010) CC2540 2.4-GHz Bluetooth R Low Energy System-on-Chip. http://www.ti.com.cn/cn/lit/ds/symlink/cc2540.pdf 